\title{
A Strong Hot Spot Theorem
}

\section{David H. Bailey and Daniel J. Rudolph}

2 May 2003

In [3] Bailey and Richard Crandall established normality base $b$ for the class of constants

$$
\alpha_{b, c}=\sum_{k=1}^{\infty} \frac{1}{c^{k} b^{c^{k}}},
$$

where the integer $b>1$ and $c$ is odd and co-prime to $b$, as well as some generalizations of this class. The proof given in [3] is rather difficult and relies on several not-well-known results, including one by Korobov on the properties of certain pseudo-random sequences. Recently it has been shown that normality can be established much more easily, as a consequence of what may be called the "hot spot" theorem [1]. Here we state and prove a strong form of the "hot spot" theorem. A weaker result is given in [5, pg. 77], and is proven by an ergodic theory argument in [2].

In the following, $\{\cdot\}$ denotes fractional part as before, and $\#[\cdot]$ denotes count. $\mu$ and $\nu$ denote probability measures on $U$ (the unit interval mod 1). $A-B$ denotes the set of $x \in A$ and $x \notin B$, and $A \Delta B=(A-B) \cup(B-A)$. The notation a.e. $x[\mu]$ means for all $x \in U$ except for a set $Q$ with $\mu(Q)=0$. A Vitali covering of a measurable set $A \subset U$ is a collection of open intervals with the property that every $x \in A$ is contained in infinitely many, arbitrarily small intervals in the collection. The measure $\nu$ is absolutely continuous with respect to $\mu$ if $\nu(A)=0$ whenever $\mu(A)=0$. The map $T: U \rightarrow U$ is said to be measure-preserving with respect to $\mu$ if $\mu\left(T^{-1} A\right)=\mu(A)$ for every $\mu$-measurable set $A$, and ergodic with respect to $\mu$ if $T^{-1} A=A$ implies $\mu(A)=0$ or 1 .

Given a real constant $\alpha$ in $[0,1)$, we define here a base-b hot spot to be some $x \in[0,1)$ with the property that

$$
\liminf _{h \rightarrow 0} \liminf _{n \rightarrow \infty} \frac{\#_{0 \leq j<n}\left[\left\{b^{j} \alpha\right\} \in(x-h, x+h)\right]}{2 h n}=\infty .
$$

Another way to state this condition is this: $x$ is a base- $b$ hot spot if given any $M>0$, there is some $\delta_{M}>0$ such that for all $h<\delta_{M}$ there is some $N_{h}>0$ such that for all $n>N_{h}$, the condition $\#_{0 \leq j<n}\left[\left\{b^{j} \alpha\right\} \in(x-h, x+h)\right]>2 h n M$ holds.

What we shall establish below is that $\alpha$ is $b$-normal if and only if it has no base- $b$ hot spots. We first present a few preliminary results.

Lemma 1 Vitali covering lemma. If a $\mu$-measurable set $A \subset U$ has a Vitali covering, then given any $\epsilon>0$, there is some finite disjoint subcollection $A^{\prime}$ with the property that $\mu\left(A \Delta A^{\prime}\right)<\epsilon$.

This result is proven in [6]. 
Lemma 2 Birkoff ergodic theorem. Let $f(t)$ be an integrable function on $[0,1)$, and let $T$ be an ergodic transformation for $\mu$. Then

$$
\lim _{n \rightarrow \infty} \frac{1}{n} \sum_{k=0}^{n-1} f\left(T^{k} x\right)=\int f d \mu \quad \text { for a.e. } x[\mu],
$$

This result is proved in [4, pg. 13, 20-29].

Lemma 3 Equivalence of absolutely continuous measures. Suppose that $T$ is measure-preserving and ergodic with respect to both $\mu$ and $\nu$, and further that $\nu$ is absolutely continuous with respect to $\mu$. Then $\mu=\nu$.

Proof. Applying the ergodic theorem to $f(t)=I_{A}(t)$ (the indicator function of $A$ ),

$$
\lim _{n \rightarrow \infty} \frac{1}{n} \sum_{k=0}^{n-1} f\left(T^{k} x\right)=\int f(t) d \mu(t)=\mu(A) \quad \text { for a.e. } x[\mu] .
$$

Since $\nu$ is absolutely continuous with respect to $\mu$, the above holds a.e. $x[\nu]$ as well. Now since $T$ preserves the measure $\nu$, we can write, for $n>0$,

$$
\begin{aligned}
\nu(A) & =\int f(t) d \nu(t)=\frac{1}{n} \sum_{i=0}^{n-1} \int f\left(T^{i} x\right) d \nu(x) \\
& =\int \frac{1}{n} \sum_{i=0}^{n-1} f\left(T^{i} x\right) d \nu(x) \rightarrow \int \mu(A) d \nu=\mu(A),
\end{aligned}
$$

by the dominated convergence theorem. QED

Lemma 4 Absolute continuity of measures with finite derivatives. Suppose $\nu$ is a measure on $U$ with the property that for a.e. $x[\nu]$,

$$
\liminf _{h \rightarrow 0} \frac{\nu(x-h, x+h)}{2 h}<\infty
$$

Then $\nu$ is absolutely continuous with Lebesgue measure.

Proof. Here $\mu$ denotes Lebesgue measure on $U$, and $\nu$ denotes any measure as defined in the hypothesis. Let $A$ be any set with $\mu(A)=0$, and let $\epsilon>0$ be given. Then there exists a set $Q$ with $\nu(Q)<\epsilon$ and $M \geq 1$ such that the LHS of (6), as a function of $x$, is bounded by $M$ except on $Q$. Further, there exists some open set $A^{\prime} \supset A$ with $\mu\left(A^{\prime}\right)<\epsilon / M$. Then for every $x \in\left(A^{\prime}-Q\right)$ there exists an infinite sequence $h_{k}$, strictly decreasing to zero, such that $\left(x-h_{1}, x+h_{1}\right) \subset A^{\prime}$ and $\nu\left(x-h_{k}, x+h_{k}\right) /\left(2 h_{k}\right) \leq M+\epsilon$ for $k \geq 1$. For $x \in\left(A^{\prime} \cap Q\right)$, define $h_{k}=2^{-m-k}$, where $m$ is large enough that $\left(x-h_{1}, x+h_{1}\right) \subset A^{\prime}$. Note that in either case all of these intervals are contained within $A^{\prime}$. The collection of 
these intervals is a Vitali covering of the set $A^{\prime}$, so by the Vitali covering lemma there is a finite disjoint subcollection $A^{\prime \prime} \subset A^{\prime}$ with $\nu\left(A^{\prime}-A^{\prime \prime}\right)<\epsilon$. We can then write

$$
\begin{aligned}
\nu(A) & \leq \nu\left(A^{\prime}\right)=\nu\left(A^{\prime \prime}\right)+\nu\left(A^{\prime}-A^{\prime \prime}\right) \\
& =\nu\left(A^{\prime \prime}-Q\right)+\nu\left(A^{\prime \prime} \cap Q\right)+\nu\left(A^{\prime}-A^{\prime \prime}\right) \\
& \leq(M+\epsilon) \mu\left(A^{\prime \prime}-Q\right)+2 \epsilon \leq(M+\epsilon) \mu\left(A^{\prime}\right)+2 \epsilon \\
& \leq(M+\epsilon) \epsilon / M+2 \epsilon<4 \epsilon,
\end{aligned}
$$

which implies that $\nu(A)=0$. QED

In the following, $\mu$ will denote Lebesgue measure on $U$, and, given a real constant $\alpha \in U$ and an integer $b \geq 2, \nu$ will denote the measure defined on an interval $(c, d)$ as

$$
\nu(c, d)=\liminf _{n \rightarrow \infty} \frac{\#_{0 \leq j<n}\left[\left\{b^{j} \alpha\right\} \in(c, d)\right]}{n}
$$

Lemma 5 Ergodicity of the digit-shift transformation. The digit-shift transformation $T(x)=\{b x\}$ is measure-preserving and ergodic with respect to both $\mu$ and $\nu$.

Proof. $T$ clearly preserves Lebesgue measure. Assume for convenience that $b=2$, and suppose that $A=T^{-1}(A)$. Then note that $x \in A$ if and only if $\{x+1 / 2\} \in A$. Thus if $D=(0,1 / 2)$, then $\mu(A \cap D)=\mu(A) / 2=\mu(A) \mu(D)$. A similar equality follows for any binary rational interval $\left(j 2^{m}, k 2^{m}\right)$, and thus for any finite disjoint union of such intervals. This collection of binary rational intervals is a Vitali covering of $A$. Thus given $\epsilon>0$, there is some finite disjoint union $E$ with $\mu(A \Delta E)<\epsilon$ and $\mu(A \cap E)=\mu(A) \mu(E)$. We can then write

$$
\begin{aligned}
\left|\mu(A)-\mu^{2}(A)\right| & <|\mu(A)-\mu(A) \mu(E)|+\epsilon=|\mu(A)-\mu(A \cap E)|+\epsilon \\
& =|\mu(A)-(\mu(A)-\mu(A-E))|+\epsilon \leq 2 \epsilon
\end{aligned}
$$

Thus $\mu(A)=\mu^{2}(A)$, so that $\mu(A)=0$ or 1 as required. A similar argument applies to the measure $\nu$ as defined above. In the parlance of ergodic theory, $T$ is "mixing" with respect to both $\mu$ and $\nu$, which condition is well-known to imply ergodicity [4, pg. 12]. QED

Theorem 1 Hot spot theorem. The real constant $\alpha$ is b-normal if and only if it has no base-b hot spots.

Proof. If $\alpha$ has no base- $b$ hot spots, then it follows immediately from Lemmas 3,4 , and 5 , that for any interval $(c, d) \subset U$,

$$
\liminf _{n \rightarrow \infty} \frac{\#_{0 \leq j<n}\left[\left\{b^{j} \alpha\right\} \in(c, d)\right]}{n}=\mu(c, d)=d-c
$$


This result also applies to $(0, c) \cup(d, 1)$, which except for $c, d$ and the point 0 is the complement of $(c, d)$. We can then write

$$
\begin{aligned}
\limsup _{n \rightarrow \infty} \frac{\#_{0 \leq j<n}\left[\left\{b^{j} \alpha\right\} \in(c, d)\right]}{n} & =1-\liminf _{n \rightarrow \infty} \frac{\#_{0 \leq j<n}\left[\left\{b^{j} \alpha\right\} \in(0, c) \cup(d, 1)\right]}{n} \\
& =1-(c+(1-d))=d-c
\end{aligned}
$$

Thus the liminf and the limsup are identical. Since this holds for any interval $(c, d)$, it holds in particular for any interval whose endpoints are of the form $j / b^{m}$. Thus $\alpha$ is b-normal. QED

\section{References}

[1] David H. Bailey, "A Hot-Spot Proof of Normality for the Alpha Constants," manuscript, 2003, available at the URL http://www.nersc.gov/ dhbailey/dhbpapers/alpha-normal.pdf

[2] David H. Bailey and Daniel J. Rudolph, "An Ergodic Proof that Rational Times Normal is Normal," manuscript, 2002, available at the URL http://www .nersc.gov/ dhbailey/dhbpapers/ratxnormal.pdf

[3] David H. Bailey and Richard E. Crandall, "Random Generators and Normal Numbers," Experimental Mathematics, vol. 11 (2002), pg. 527-546.

[4] Patrick Billingsley, Ergodic Theory and Information, John Wiley, New York, 1965.

[5] L. Kuipers and H. Niederreiter, Uniform Distribution of Sequences, Wiley-Interscience, New York, 1974.

[6] Halsey L. Royden, Measure Theory, Addison-Wesley, 1968. 